\title{
Kaffee als Ko-Analgetikum bei Opioidtherapie?
}

Patienten mit Fibromyalgie, die chronische Schmerzen haben und dagegen Opioide erhalten, verstärken möglicherweise den analgetischen Effekt durch Kaffeetrinken. Erstmals Hinweise darauf liefern jetzt die Ergebnisse einer Studie US-amerikanischer Rheumatologen.

Koffein als Ko-Analgetikum (- Abb. 1) wird etwa zur Selbstmedikation bei Migräne sowie beim Kopfschmerz vom Spannungstyp von der Deutschen Migräne- und Kopfschmerzgesellschaft empfohlen, und zwar in Kombination mit Acetylsalicylsäure und Paracetamol. Die Ärzte um Dr. J. Ryan Scott von der Universität von Michigan stellten nun für ihre Studie zum einen die Hypothese auf, dass Koffein einen intrinsischen analgetischen Effekt hat. Sie stützen sich dabei auf Angaben von Patienten, die regelmäßig Kaffee trinken.

Die zweite These war, dass Koffein auch bei Patienten, die Opioide einnehmen, einen koanalgetischen Effekt hat. Für ihre Querschnittsstudie wählten die Rheumatologen Fibromyalgiepatienten mit chronischen Schmerzen.

\section{$\mathbf{8 0} \%$ der Studienteilnehmer tran- ken regelmäßig Kaffee}

Von insgesamt 972 Studienteilnehmern standen die Ergebnisse unter anderem von Befragungen zum Kaffeekonsum und zum Opioidgebrauch zur

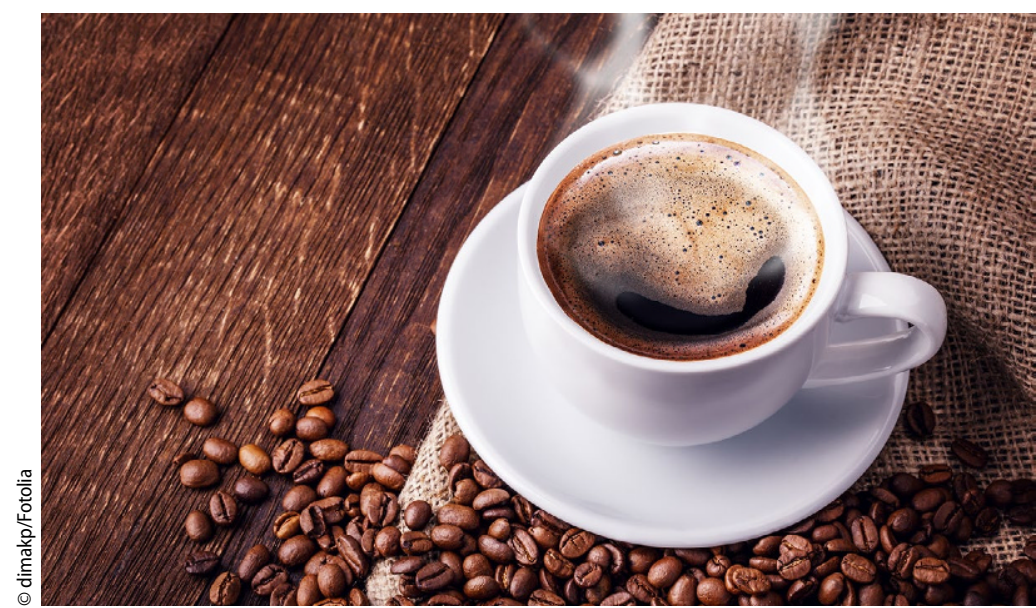

Quelle: Peter Leiner, SpringerMedizin.de

Verfügung. $67 \%$ der Patienten waren Frauen. 568 Patienten (59\%) hatten angegeben, Opioide täglich oder nach Bedarf zu nutzen. Der Anteil der Studienteilnehmer mit beziehungsweise ohne Opioidtherapie, die regelmäßig Kaffee tranken, betrug $81 \%$ bzw. $80 \%$. In den Gruppen mit und ohne Opioidtherapie unterschied sich der Anteil jener Patienten, die zur Schmerzlinderung außerdem Acetylsalicylsäure, Ibuprofen und/oder Paracetamol einnahmen, nicht wesentlich (49\% bzw. $51 \%$ ).

\section{》) Die Kaffeetrinker litten auch} seltener an Depressionen

Aus den Ergebnissen der Studie geht hervor, dass Patienten mit Opioid-Therapie, die regelmäßig Kaffeetrinken, eigenen Angaben zufolge weniger starke Schmerzen haben. Außerdem gaben sie an, dass ihre Schmerzen den Alltag weniger beeinflussten und sie die Schmerzen weniger überbewerteten als Patienten mit Opioidtherapie, aber ohne Kaffeekonsum.

Abb. $1 \Delta$ Koffein hemmt die Rezeptoren für Adenosin, das an der Schmerzentstehung und-modulation beteiligt ist
Schließlich litten die Kaffeetrinker seltener unter Depressionen. Wie Scott und seine Kollegen berichten, konnten sie zudem einen dosisabhängigen $\mathrm{Ef}$ fekt beobachten. Allgemein betrachtet linderte ein geringer bis moderater täglicher Konsum (weniger als eine Tasse bis maximal 2,5 Tassen) unter anderem die Schmerzsymptome, hoher Konsum (mehr als drei bis zwölfTassen) dagegen nicht.

\section{Kausaler Zusammenhang nicht ab- leitbar}

Die Rheumatologen weisen einschränkend darauf hin, dass sich aufgrund des Studiendesigns kein kausaler Zusammenhang zwischen Kaffeekonsum zusätzlich zur Opioidtherapie und Schmerzlinderung ableiten lässt. Außerdem sei mit einer Verzerrung der Ergebnisse zu rechnen, weil die Patienten möglicherweise zu geringe Angaben über den tatsächlichen Schmerzmittelgebrauch gemacht hätten. Schließlich fehlten exakte Informationen zur aufgenommenen Koffein- und Opioidmenge.

Der Wirkmechanismus von Koffein auf die Schmerzwahrnehmung ist nach Angaben der Ärzte komplex und noch nicht im Einzelnen verstanden. Bekannt ist, dass es die Rezeptoren für Adenosin hemmt, das an der Schmerzentstehung und-modulation beteiligt ist.

\section{Literatur}

1. Scott JR et al (2017) Caffeine as an opioid analgesic adjuvant in fibromyalgia. J Pain Res 10:1801-1809. http://dx.doi.org/10.2147/ JPR.S134421

rheuma plus $2017 \cdot 16: 118$ https://doi.org/10.1007/s12688-0170135-1

○ Springer-Verlag GmbH Austria 2017 